\title{
La transmission écrite du coran dans les débuts de l'islam. le codex parisino-petropolitanus
}

Leiden - Boston, Brill (« Texts and Studies on the Qur'ān », 5), 2009, IX 208 - 383 p. [ISBN 978900417272 2]

\section{Alba Fedeli}

\section{CpenEdition}

\section{Journals}

Édition électronique

URL : http://journals.openedition.org/beo/209

DOI : $10.4000 /$ beo. 209

ISBN : 978-2-35159-318-9

ISSN : 2077-4079

\section{Éditeur}

Presses de l'Institut français du Proche-Orient

\section{Édition imprimée}

Date de publication : 1 octobre 2010

Pagination : 149-157

ISBN : 978-2-35159-170-3

ISSN : 0253-1623

\section{Référence électronique}

Alba Fedeli, «La transmission écrite du coran dans les débuts de l'islam. le codex parisinopetropolitanus », Bulletin d'études orientales [En ligne], Tome LIX | octobre 2010, mis en ligne le 01 octobre 2011, consulté le 21 septembre 2020. URL : http://journals.openedition.org/beo/209 ; DOI https://doi.org/10.4000/beo.209

Ce document a été généré automatiquement le 21 septembre 2020

(c) Institut français du Proche-Orient 


\section{La transmission écrite du coran dans les débuts de l'islam. le codex parisino-petropolitanus}

Leiden - Boston, Brill (« Texts and Studies on the Qur'ān », 5), 2009, IX 208 - 383 p. [ISBN 978900417272 2]

\section{Alba Fedeli}

\section{RÉFÉRENCE}

La transmission écrite du coran dans les débuts de l'islam. le codex parisinopetropolitanus. Leiden - Boston, Brill (« Texts and Studies on the Qur'ān », 5), 2009, IX 208 - 383 p. [ISBN 978900417272 2]

1 Ce livre représente une synthèse - à partir de l'analyse du codex Parisinopetropolitanus - des idées développées par l'auteur sur la transmission écrite du Coran dans les débuts de l'islam, à laquelle il a consacré depuis longtemps tous ses efforts et d'innombrables études. L'analyse, qui met en évidence la complexité et l'originalité du codex du Coran et du travail des copistes qui ont transcrit le texte, nous semble une captivante et piquante réplique au lieu commun erroné selon lequel les manuscrits coraniques sont identiques : 208 pages d'analyse du codex P.P. et 383 pages dédiées à la transcription de son texte le démontrent d'une manière manifeste, dans l'ouvrage ici recensé.

2 L'analyse exhaustive de ce seul codex coranique nous évoque le récit que François Déroche a relaté à propos de son travail de catalogage des manuscrits coraniques de la Bibliothèque nationale de France, publié en $1983 .{ }^{1} \mathrm{~A}$ l'occasion de la Conférence internationale sur les manuscrits du Coran organisée à Bologne en 2002, F.D. a introduit les travaux en montrant la situation de l'étude des manuscrits coraniques entre passé et avenir. ${ }^{2}$ Il a mentionné un épisode emblématique de l'orientation suivie dans ce domaine, qui nous semble important de souligner, au-delà de l'anecdote. Avant de 
commencer sa description des manuscrits arabes 324-589 de la Bibliothèque nationale, à savoir la collection des manuscrits coraniques, F.D. avait demandé un renseignement à Georges Vajda à propos de l'omission des ces derniers dans le travail de catalogage des manuscrits arabes musulmans de la Bibliothèque, publié en $1978^{3}$. G. Vajda expliqua qu'il avait consacré ses efforts à la littérature arabe, et estima que la description de près de 600 pièces dont le texte est identique aurait été un gaspillage de temps et d'énergie. ${ }^{4}$

3 La richesse et l'importance des résultats que F.D. a soulignées dans son ouvrage, prouvent que la description du codex P.P. n'a pas été un «gaspillage de temps et d'énergie ». L'auteur continue son récit : «J'ai tout de suite découvert que ce matériau était beaucoup plus riche mais aussi beaucoup plus difficile que l'opinion de Vajda avait suggéré $» .^{5}$ Deux pièces parmi les manuscrits de la BnF, à savoir les manuscrits arabes $328 \mathrm{a}$ et $328 \mathrm{~b}$ - que l'auteur a longuement fréquenté - faisaient partie, avec d'autres fragments, d'un unique codex de quatre-vingt huit feuillets conservés, actuellement disloqués en quatre collections, à Paris justement (soixante-dix ff.), Saint-Pétersbourg (vingt-six ff.), Londres (un feuillet) et Vatican (un feuillet), appelé dès lors codex Parisino-petropolitanus. Après une reconstruction virtuelle, le codex a été analysé au niveau paléographique, codicologique et philologique, en essayant de reconstituer l'histoire du codex et de ses pérégrinations ainsi que les modalités du travail des copistes par rapport au modèle du texte aux débuts de l'islam.

4 L'ouvrage représente en partie, comme l'auteur le définit, une palinodie : en effet F.D. re-visite d'abord ses critères de distinction entre le ms arabe $328 \mathrm{a}$ et $328 \mathrm{~b}$ et ses conclusions exposées dans le catalogue de 1983, à la lumière des informations recueillies grâce à l'étude, à partir de 2001, de la série conservée à Saint-Pétersbourg et à la nouvelle reliure du manuscrite arabe 328 dans le 2006 qui a permis à l'A. de reconnaître la même provenance des deux fragments alors distingués comme arabe $328 \mathrm{a}$ et $328 \mathrm{~b}$.

L'évolution prometteuse que les recherches sur les manuscrits coraniques connaissent depuis quelques années, est manifestée par la publication de deux ouvrages dédiés au manuscrit arabe 328a de la BnF : en 2009 précisément a été publié un autre ouvrage qui a comme point de départ l'analyse du texte du fragment du codex qui se trouve à la $\mathrm{BnF}$, même si l'approche est complètement différente. Il s'agit du livre de David S. Powers Muhammad is not the father of any of your men. The making of the last Prophet. ${ }^{6}$ Powers n'a pas considéré dans son ensemble le texte coranique tel qu'il est transmis dans le manuscrit arabe $328 \mathrm{a}$ et $328 \mathrm{~b}$ de la BnF ; mais il a utilisé seulement l'analyse de deux passages coraniques que le parchemin a conservés, soit les deux récurrences du mot kalāla (Q.4:12 et 176) afin de corroborer une thèse précédemment formulée. En 1982, Powers avait déjà publié une étude au sujet d'une nouvelle lecture du verset 12 de la sūrat al-nisā' et une nouvelle interprétation des dispositions testamentaires selon le Coran qui en dériverait. ${ }^{7}$ Dans cette étude de 1982 il suggérait que le Coran avait admis le droit pour un homme de nommer comme son unique héritière une femme avec laquelle il est apparenté sans aucun lien du sang. Dans son ouvrage, Powers se limite à l'analyse de la correction du mot *kalla en kalāla, dès lors que le but de son étude était la théorie sur l'abolition de l'adoption dans l'islam, en oubliant une contextualisation de cette correction par une compréhension de la signification des autres modifications apportées à la première écriture du manuscrit dans leur comparaison. Par contre dans l'ouvrage de F.D., le codex P.P. - considéré dans toute son étendue - a été l'objet d'une 
analyse méticuleuse sans procéder à l'examen des conséquences de certaines corrections au niveau du signifié. ${ }^{8}$

6 Les manuscrits coraniques de la BnF en général et l'arabe 328 en particulier sont très célèbres. Amari les a étudiés en écrivant au terme de son parcours un ouvrage ${ }^{9}$ qui reçut le prix ex aequo avec le jeune Nöldeke et Sprenger, accordé par l'Académie des Inscriptions et belles-lettres, concernant l'histoire du Coran..$^{10}$ Plus récemment, d'après la publication du fac-similé du manuscrit, ${ }^{11}$ Dutton a publié en 2001 un article sur l'analyse de son texte. ${ }^{12}$ Néanmoins l'ouvrage de F.D. est une avancée innovante, en comblant une lacune des études des anciens manuscrits coraniques.

7 L'auteur divise l'ouvrage entre deux parties: la première est consacrée à la transmission écrite du Coran en analysant le codex P.P., alors que dans la deuxième partie il propose une transcription de l'ensemble du texte. Avant de procéder à l'analyse proprement dite du codex, dans le premier chapitre (« Les pérégrinations d'un manuscrit ») l'auteur retrace l'histoire fascinante et encore partiellement mystérieuse du codex à partir de ses premiers vestiges évoqués dans la mosquée de Fusțāț un des très importants anciens dépôts de manuscrits coraniques, en décrivant le milieu de chercheurs de manuscrits dans l'Egypte du début du dix-neuvième siècle et les pérégrinations de manuscrits, qui n'ont jamais cessées, vu que «des feuillets isolés circulent peut-être encore »(p.18).

Le deuxième chapitre («Analyse codicologique du codex Parisino-petropolitanus ») est consacré à l'étude de la constitution matérielle du manuscrit. Une fois la structure codicologique décrite, à savoir les quaternions constitués par assemblage de bifeuillets et qui représentent une structure très homogène, ${ }^{13}$ l'auteur consacre la deuxième partie du chapitre à l'analyse paléographique du manuscrit qui a vu la collaboration de cinq copistes qui ont travaillé en respectant des règles générales et en se relayant pour assurer la continuité de la copie du texte, présenté par double page (p. 28-29). ${ }^{14}$ Cette " entreprise collective» (p.28) est l'une des caractéristiques les plus marquantes du codex, pour une reconstruction de la méthode de travail des copistes et de leur importance et autonomie : si chacun d'entre eux a sa spécificité, ils suivent cependant des règles générales établies, en donnant une identité visuelle aux pages du codex. Certains éléments de cette identité visuelle sont communs à tous les manuscrits en style hiğ $\bar{a} z \bar{i}$, comme récemment Alain George a noté dans sa lucide description des origines de l'écriture arabe. ${ }^{15} \mathrm{~F}$.D. détermine les traits distinctifs de chaque copiste (les mains A, B, C, D et E), en analysant l'exécution de certaines lettres "caractéristiques » et l'emploi des diacritiques. Ensuite l'auteur étudie les interventions - grattages et ajouts - qui se trouvent dans le texte, en distinguant les erreurs rectifiées et décelées sans retard par le scribe et les retouches d'intervenants postérieurs en ce qui concerne le texte coranique et, d'autre part, les retouches apportées aux divisions du texte. Les interventions qui appartiennent à des phases successives de l'histoire du texte ont été utilisées par l'auteur comme éléments pour une datation de l'utilisation du codex P.P.

9 L'auteur développe ensuite dans le troisième chapitre l'orthographe du codex P.P., en s'efforçant d'écarter les corrections postérieures et utilisant comme point de départ l'édition égyptienne de 1932, bien que cette édition ne soit pas destinée à des philologues et implique "des inconsistances »: l'auteur en est conscient et nous en avertit dès le début du chapitre (p. 52-53). Après cet avertissement, F.D. développe l'analyse de l'orthographe de cinq racines, dont les occurrences sont nombreuses dans le Coran, en soulignant la spécificité de chaque copiste, qui a suivi des options 
individuelles. L'auteur y propose une des conclusions les plus importantes de son ouvrage, à savoir la distinction entre règles et options dans le travaille des scribes: «L'analyse des seules formes prises par ces cinq racines a déjà fait apparaitre que les solutions adoptées varient selon les copistes du manuscrit. [...] La relative homogénéité observée dans chaque cas invite à supposer qu'il s'agissait d'options prises par les copistes et non de variations d'un éventuel original» (p.56). Il souligne encore que « les cinq copistes avaient adopté (ou conservé) en matière d'orthographe des solutions personnelles et n'avaient visiblement pas cherché à définir une position commune qui aurait pu être maintenue du début à la fin du texte coranique » (p. 59). La même conclusion est proposée dans la dernière partie du chapitre, dans laquelle, après avoir analysé les divergences dans la notation du /ā/, l'utilisation du y $y \bar{a}$ pour l'indiquer, l'utilisation contrastée de l'alif al-wiqāya et les intermittences dans la notation de la présence d'un hamza, l'auteur souligne le « caractère éminemment personnel, presque spontané, de l'orthographe des copistes du Parisino-petropolitanus» (p.74), en présentant l'absence de règles dans l'orthographe ancienne de la langue coranique et en mettant en évidence l'autonomie des copistes avec leur " pratiques récurrentes » et leur « habitudes ", en cherchant d'améliorer le rasm.

10 L'analyse de la constitution matérielle du manuscrit et de son orthographe étant donnée, l'auteur procède à l'étude du texte du codex P.P., notamment à l'étude de la division du texte coranique en versets et des «variantes » du rasm, exception faite des variations de l'orthographe, que l'A. ne considère pas comme "variantes ». D'abord l'auteur donne une description de séparations des versets, en distinguant les caractéristiques de chaque copiste et en séparant la première intervention, contemporaine de la copie du texte, de deux interventions postérieures, y comprenant les grattages. La comparaison des fins des versets marquées dans le manuscrit avec les divisions conservées par la tradition montre que le codex représente une tradition propre, qui constitue un autre élément pour en déduire la complexité du texte transmis. Une fois cette analyse développée, à partir du travail de Anton Spitaler, l'auteur distingue les informations sur la division des versets qui ont été données au lecteur après l'achèvement de la copie, en proposant l'existence de trois phases : celle du copiste, contemporaine à la copie ; celle du rubricateur, qui a marqué chaque groupe de cinq et dix verset à l'encre rouge et que l'auteur propose de placer avant la fin du $\mathrm{II}^{\mathrm{e}} / \mathrm{VII}^{\mathrm{e}}$ siècle, et enfin celle du numéroteur, qui a utilisé des lettres-chiffres à l'encre noire, probablement au cours du III $/ \mathrm{IX}^{\mathrm{e}}$ siècle. Au terme de ce parcours, l'auteur conclut en soulignant encore une fois l'histoire du manuscrit et son importance " qui justifie que jusque dans le courant $\mathrm{du} \mathrm{III}^{\mathrm{e}} / \mathrm{IX}^{\mathrm{e}}$ siècle, probablement dans sa deuxième moitié, on y ait apporté des retouches » (p. 101). L'analyse du texte est achevée en y dégageant ses variantes, à partir des résultats de l'analyse de Dutton publiée en 2001, bien qu'elle soit partielle et limité aux feuillets 1-56 du ms arabe 328a de la BnF. Il s'agit pour l'essentiel de variantes canoniques, sauf « une dizaine de situations ", que l'on ne peut pas élucider par les particularités orthographiques du manuscrit ni par les erreurs des copistes.

11 L'auteur a achevé dans les premiers chapitres une analyse, qui est l'aboutissement d'une investigation et vérification directe du manuscrit original afin d'écarter les limites d'un examen de ses reproductions. L'auteur souligne ces limites dans l'étude de Dutton, qui a travaillé sur le fac-similé et de ce fait, à titre d'exemple, "n'a pas tenu compte de ces différentes phases, bien qu'il ait entrevu le problème » (p. 79, n. 4) en ce qui concerne les différentes phases de la numérotation des versets et « en travaillant 
sur le facsimilé, il n'a pas toujours identifié ces marques de versets grattées » (p. 80, n. 8). Ainsi, l'étude du codex a été menée par F. D. selon une double perspective. Il a basé son examen et ses conclusions - dans les chapitres consacrés à l'analyse de la constitution matérielle du manuscrit, de son orthographe et de son texte - sur une comparaison du texte du manuscrit tout d'abord avec le texte de l'édition égyptienne de 1932 et deuxièmement avec la littérature traditionnelle. Il s'agit de décrire le texte transmis par rapport au texte standard d'une part, ainsi que de déterminer, d'autre part, le rapport réciproque entre ces deux et le cadre des informations transmises par la littérature traditionnelle.

12 L'auteur a tenu compte du texte de l'édition égyptienne par nécessité, bien qu'il soit conscient de la relativité de cette "norme». On remarquera avec l'auteur, que, par rapport à l'analyse de son orthographe, «les comparaisons sont souvent compliquées par les incohérences de l'édition du Caire » (p.69) et que à propos de cette base de la comparaison « on notera qu'elle présente sur ce point quelques inconsistances » (p. 71). A propos des critères personnels appliqués par les copistes, "de manière plus systématique que dans l'édition du Caire» (p. 73), ils faisaient preuve «d'une plus grande cohérence que les savants égyptiens responsables de l'édition du Caire, sans l'être jamais totalement " (p. 75). De plus, l'usage du manuscrit pendant des siècles et les modifications qui y furent apportées sont preuves d'une norme variable, comme l'auteur souligne : « le Parisino-petropolitanus a donc été soumis à une suite de lectures et de contrôles qui ont visé à en faire disparaître les fautes ou les divergences par rapport à une norme qui a pu varier au fil du temps» (p. 105 - 106). Les chercheurs sont toujours implicitement soumis à la norme en vigueur à leur époque; en effet, comme souligne F.D., quand Amari avait étudié les manuscrits de la collection de la BnF, il observa que «l'orthographe de ces manuscrits doit nous intéresser plus encore, écrivait-il, que leur calligraphie ; en la comparant à l'orthographe actuelle, on trouve des anomalies considérables » (p. 18). Dans la note de bas de page, F. D. remarque que par orthographe actuelle, il faut entendre celle de l'édition de Hinckelmann de 1694 (p. 18, n. 50 et p. 52, n. 5).

13 En ce qui concerne la comparaison des variantes du codex avec la littérature traditionnelle, l'auteur consacre une brève partie (p. 102 - 108) de son analyse, en utilisant les données de l'étude de Dutton relativement aux 56 feuillets du ms 328a, rattachés à la lecture d'Ibn 'Āmir. Afin de repérer les variantes vérifiées dans le codex, F.D. se reporte seulement à l'ouvrage d'al-Dānī, notamment le Kitāb al-Muqni 'ì ma'rifa marsūm mașāhif ahl al-amșār, dans les deux éditions de M.A.Dahmān et de Otto Pretzl, ainsi qu'à la troisième partie de la Geschichte des Qorâns ${ }^{16}$ et à l'ouvrage de Ibn Abī Dāwūd, Kitāb al-mașāhif, édité par Arthur Jeffery. Les neuf passages du texte non analysés par Dutton et qui sont signalés parmi les variantes de la tradition, sont communs à Médine et Damas, alors que « une seule variante, en $57: 10$, constitue une véritable spécificité de la lecture d'Ibn ‘Āmir» (p. 104). L'auteur souligne l'intérêt des variantes non canoniques (ou plutôt les «autre variantes»), à savoir les «lectures spécifiques dont nous pouvons accepter à titre d'hypothèse qu'elles reflètent une tradition dont le souvenir s'est perdu » (p. 108). Il s'agit en effet des variants perçues comme différentes, dans la période suivante, quand « les correcteurs ont en tout cas jugé son texte suffisamment différent pour entreprendre de l'amender et de le rapprocher » (p. 108) de la vulgate 'uțmānienne. 
14 La méthode utilisée par l'auteur pour examiner le codex est abordée dans le cinquième et dernière chapitre ( Le codex Parisino-petropolitanus et les débuts de la tradition coranique manuscrite »). Le codex ne peut en effet être abordé que lorsqu'on a tracé sa contextualisation, en mettant en relation le codex - sa forme et son contenu - avec les autres manuscrits que l'on peut attribuer à la même période, en ayant les mêmes traits distinctifs. Ce chapitre se divise en quatre parties. Dans la première, avant de procéder à l'analyse des autres manuscrits, F. D. essaie de résoudre le débat qui a surgi autour de la définition d' "écriture de style hiğăzī », qui est souvent considérée un «scholarly artefact », mais qui, selon l'auteur, est justifiable dès qu'on applique ce qualificatif «non pas une écriture, mais à un style dont le dénominateur commun est représenté par l'alif» (p. 114), en utilisant donc les termes au pluriel : écritures, en se référant à la célèbre forme de Michele Amari, « écriture du Hedjâz ».

Dans la partie suivante («La codicologie »), l'auteur illustre le corpus des manuscrits utilisés pour une comparaison avec le codex P.P., notamment

16 - le ms de Londres: British Library or. 2165, qui provient du même exemplaire que Koweït (LNS 63 MS e) et du BnF ar 328 e ;

17 - les ms de Paris : ar 328 c, 328 e, 328 f et 6140 a, ce dernier provenant du même exemplaire que Cambridge, Add. 1125 ;

18 - les ms de Saint-Pétersbourg: Marcel 17 ; Marcel 19 qui provient du même exemplaire que BnF $328 \mathrm{f}$;

19 - les ms de Sanaa, Dār al-MaHțūṭāt : 01-14.8, 01-25.1 et 01-27.1;

20 - les ms d'Istanbul, Musée des arts turc et islamique : les « Papiers de Damas », autour desquels l'auteur ne donne pas ultérieures précisions, sauf qu'il s'agit de «quarante regroupements de feuillets» (p. 124-125) et qu'on peut distinguer parmi eux deux typologies, les manuscrits de grand format et les petits manuscrits.

21 L'auteur informe le lecteur qu'il n'a pas pris en considération les fragments qui ne contiennent pas une portion de texte suffisante pour autoriser quelques comparaisons avec le P. P.; non plus que le palimpseste de Agnes Smith Lewis (Cambridge University Library Or. 1287), le ms de Cambridge (Cambridge University Library, Add. 1125) qui provient du même exemplaire que BnF ar 6140 et le ms de Birmingham (Selly Oak Colleges Library 1572), de même origine que BnF ar 328e (p. 120, note 31). Nous n'avons pas observé cette ressemblance entre ces deux manuscrits. Selon nous le manuscrit de Birmingham, qui est accessible sur le site on-line de la bibliothèque de Birmingham dans la «Virtual Manuscript Room» (http://vmr.bham.ac.uk), a été réalisé par deux copistes : il appartient en partie (ff. 1 et 7) au même exemplaire que BnF 328c et nous pourrions supposer que il s'agit partiellement (ff. 2 - 6 et 8 - 9) de la même copie que Marcel 17, bien qu'il soit un hypothèse fondée seulement sur la reproduction en blanc et noir publiée dans l'ouvrage ici recensé. La ressemblance proposée par F. D. entre le ms Selly Oak Colleges Library 1572 et BnF ar 328 e est évidemment un lapsus calami. En décrivant les manuscrits du corpus, l'A. associe le ms de Londres (p. 121) seulement au ms BnF ar 328 e et non au ms de Birmingham, qui conséquemment n'est pas associable ni au ms de Londres ni au ms BnF ar 328 e.

22 Le corpus pour une contextualisation du codex P.P. est - bien que très succinctement analysé selon la même méthode utilisée pour le P. P. dans les chapitres précédents, à savoir une analyse au niveau codicologique et un examen des écritures utilisées, résultat de la collaboration entre plusieurs copistes (dans la conclusion de la deuxième 
partie du chapitre) et au niveau du texte, comprenant les caractéristiques textuelles des manuscrits, à savoir l'orthographe, les divisions en versets et les variantes du texte (dans la troisième partie du chapitre). Bien que l'auteur ait informé le lecteur qu'il a choisi « un petit groupe de manuscrits et de fragments contenant une portion de texte suffisante pour autoriser quelques comparaison avec le Parisino-petropolitanus » (p. 119), la comparaison est inévitablement très concise et condensée et, en considérant l'étendue de l'ouvrage, dépourvue de caractère systématique par rapport aux éléments soulignés dans l'analyse du codex P.P. De plus le critère de la longueur des fragments choisis à fin de contextualisation, est apparemment en contraste avec certaines affirmations de l'auteur, par exemple en ce qui concerne les fragments de Sanaa «sur lesquels notre information demeure trop fragmentaire » (p. 144).

$\mathrm{Au}$ final, dans la quatrième partie du chapitre ( le Parisino-petropolitanus: conclusions »), l'auteur achève ses intéressantes et nouvelles conclusions sur le codex P.P., en les mettant en relation avec le contexte culturel et historique dans lequel il a été copié et utilisé au fur et à mesure. En premier lieu l'auteur souligne la présence d'un modèle qui s'était imposé en donnant aux manuscrits coraniques en style hiğ̄āzi une identité visuelle. En outre F.D., sur la base de la typologie des erreurs corrigées et des interventions sur le texte, reconstruit l'histoire du codex P.P., qui porte "tous les signes d'une longue utilisation durant la période abbasside» (p. 158). Enfin, l'auteur souligne encore une fois l'importance du codex P. P. dans les conclusions de son ouvrage, en conciliant une méthode de type état de la question (la transmission écrite du Coran dans les débuts de l'islam) et une ouverture vers des nouveaux questionnements et de nouvelles réponses, à travers l'examen concret du codex P.P., étudié sous toutes ses perspectives par la confrontation avec le texte standard et les autres manuscrits.

Tel est le plan général de l'ouvrage, suivi par un utile résumé en anglais, un index de manuscrits et de noms propres mentionnés. L'auteur a pourvu son ouvrage de précieuses planches qui donnent au lecteur les indispensables reproductions, malheureusement blanc et noir, de certains feuillets des manuscrits mentionnés dans l'analyse, ainsi que des tableaux corres-pondants des formes caractéristiques des lettres. Dans le texte (p.123) il renvoie à la planche 15 relativement au ms Marcel 19, alors que l'exemple reproduit dans la planche 15 est le $\mathrm{ms} B n F$ ar $328 \mathrm{f}$, qui est de la même origine que le ms Marcel 19. Parmi les exemples, l'auteur introduit aussi une page du palimpseste vendu par Sotheby's le 22 octobre 1992 (planche 17, p. 201), alors que dans l'analyse (p. 124) il renvoie à la planche 17 comme exemple du palimpseste de Sanaa (01-27.1) en présupposant, sans l'expliquer au lecteur, que le feuillet de Sotheby's appartient au même exemplaire du ms 01-27.1.

Après la partie de l'analyse, l'ouvrage est complété par une transcription du texte du codex P. P., qui suit la numération en chiffres indiens. L'auteur, qui a choisi de donner une transcription et non une édition du texte, fait précéder cette dernière d'un avertissement court et condensé (p. r), en indiquant les exceptions par rapport au manuscrit original et les signes introduits pour marquer le début de page du codex, le texte mutilé, les versets ajouté et les grattages. En effet l'auteur développe tous les éléments pour introduire la transcription seulement dans son analyse. Pour la transcription F.D. avertit le lecteur qu'il n'a pas repris la ponctuation diacritique moderne, en proposant une transcription du texte originel du manuscrit, plutôt que la transcription de l'édition du Caire. Néanmoins l'auteur y a uniquement apporté des 
modifications (p. 4), en supprimant certaines éléments des caractères utilisés, par exemple en ce qui concerne la forme du kāf final : il a dépouillé la lettre de l'édition du Caire de son hamza, en obtenant une forme qui est ambiguë et qui en même temps ne reproduit aucun trait de la lettre en style hiğăzi. D'autre part cette transcription, à savoir l' « égyptienne » modifiée, n'est pas proposée dans l'analyse. Je ne me réfère pas à l'inévitable option de restituer "lors de la lecture» les points diacritiques en se conformant au texte du Caire (p. 53); il s'agit plutôt des cas dans lesquels l'auteur a ajouté des points diacritiques qui ne se trouvent ni dans le texte original du manuscrit ni dans la vulgate. On peut lire par exemple bayanāt dans l'analyse (p. 63), avec les بينيت points diacritiques pour marquer le dernier $y \vec{a} \bar{a}^{\prime}$, qui n'existe pas évidemment dans l'édition du Caire (بينت), en manifestant les traits caractéristiques de l'orthographe de la période et du manuscrit, alors que dans la transcription on lit بينت . Quelle est la lecture de l'auteur alors que la notation de la voyelle longue $\bar{a}$ par moyen d'un $y \bar{a}$ ' sans points diacritiques n'existe pas dans l'édition du Caire?

La question de la conformité du manuscrit avec la vulgate 'uțānienne est d'autre part un des éléments les plus intéressants que l'auteur trace selon un point de vue innovateur. L'auteur, à travers l'examen concret et rigoureux d'un codex complexe, propose une réponse concrète au sujet du rapport des manuscrits avec un exemplar. La présentation matérielle des manuscrits en style hiğăzī qui s'imposent comme identité visuelle s'explique "par la volonté d'imposer une version qui avait reçu l'aval du pouvoir » (p. 162) et conforte "l'hypothèse de l'existence d'un modèle revêtu d'une autorité certaine, un exemplaire initial qui aurait constitué une référence incontestable » (p. 162), même en admettant la présence d'une " entreprise de copie », marquée par les options personnelles des copistes et en constatant que la tradition manuscrite est encore insuffisamment codifiée à cette époque. C'est l'histoire même de l'édition du calife 'Uțmān qui «demande à être reconsidérée à la lumière de ces témoins primitifs» (p. 163-164), en dépassant l'approche contraire, à savoir la lecture des manuscrits à la lumière de l'histoire de l'édition du calife 'Uțmān. Une fois de plus, il est montré que les études coraniques ont beaucoup à gagner de l'analyse des manuscrits, « les seuls vestiges susceptibles de s'approcher le plus près des origines ", pour « confronter ce que rapportent les sources et les vestiges matériels eux-mêmes » (p. 3).

27 Au final, cet ouvrage s'impose comme une référence dans les études des manuscrits coraniques en style hiğăzī, en souhaitant qu'il soit le premier d'une nouvelle collection sur "La transmission écrite du Coran dans les débuts de l'islam». On remarquera d'autre part avec l'auteur, que «l'orthographe ancienne de la langue coranique est complexe et présente encore bien des obscurités que l'étude d'un nombre plus important de manuscrits contemporains du Parisino-petropolitanus permettra sans doute d'élucider " (p. 75). Nous étendons néanmoins cette affirmation à toutes les typologies des variantes vérifiées dans les manuscrits. Les doutes manifestés par F. D. au sujet des "variantes inconnues par ailleurs ", en se demandant « Mais convient-il de leur reconnaitre ce statut? Ne pourrait-il pas s'agir de fautes de copistes?» (p. 145), pourraient avoir une réponse par une comparaison entre la totalité du corpus des anciens manuscrits. Afin de pouvoir reconnaitre une faute du copiste en la distinguant d'une "variante", l'étude du corpus complet des manuscrits en style hiğāāi et leur comparaison s'impose: la présence récurrente d'un mot pourrait nous aider à distinguer entre fautes et variantes, entre variantes et variantes établies sur des vestiges. Toutefois un tel projet requiert une collaboration entre plusieurs institutions 
et spécialistes, comme Blachère souhaitait et suggérait à propos de ce qu'il appelait " édition critique du texte du Coran », dans son introduction au Coran en 1958 : «Une œuvre de cette ampleur nécessite une collaboration internationale, une mis en commun de toutes les ressources en manuscrits existant dans le monde ».17

\section{NOTES}

1. DÉROCHE François, Les manuscrits du Coran. Aux origines de la calligraphie coranique, Paris, Bibliothèque Nationale, 1983. (Catalogue des manuscrits arabes. Deuxième partie : Manuscrits musulmans, tome I, 1).

2. DÉROCHE François, "Studying the Manuscripts of the Qur'ān, Past and Future", Mélanges de l'Université Saint-Joseph, vol. 59, 2006, (Actes de la Conférence internationale sur les manuscrits du Coran, Bologne, 26-28 septembre 2002), p. 163-181.

3. VAJDA Georges - SAUVAN Yvette, Manuscrits musulmans, Paris, Bibliothèque Nationale, 1978. (Catalogue des manuscrits arabes. Deuxième partie: Manuscrits musulmans, tome II, $\mathrm{N}^{\text {os }}$ 590-1120).

Dans son avant-propos (p. XI, note 2) Vajda précisa au sujet de l'omission des manuscrits du Coran dans son catalogue que « la raison de cette omission est qu'une description adéquate des manuscrits et fragments du Coran en caractères coufiques ( $\mathrm{n}^{\text {os }} 324-383,5122-5124,5178-5179$, $6002,6087,6140,6430,6982)$ avait pour condition préliminaire des recherches d'ordre paléographique et codicologique sur les matériaux en question; entamées, il y a un certain nombre d'années, ces recherches n'ont pu, malheureusement, être menées à bien ».

4. DÉROCHE, "Studying the manuscripts",p.163.

5. DÉROCHE, "Studying the manuscripts",p. 163-164.

6. POWERS David S., Muhammad is not the father of any of your men. The making of the last Prophet, Philadelphia, University of Pennsylvania Press, 2009.

7. POWERS David S., "The Islamic law of inheritance reconsidered: a new reading of Q.4:12b", Studia Islamica, vol. 55, 1982, p. 61-94.

8. Au sujet des corrections apportées dans le verset 12 de la sūrat al-nis $\bar{a}$, F.D. souligne (p.107) « وa-lahā au lieu de وله wa-lahuest la seule portion déchiffrable, quoique retouchée, d'un passage qui a été soigneusement effacé ", en renvoyant dans une note de bas de page à l'ouvrage de Powers : «Ce passage du manuscrit fait l'objet d'une étude de D. S. Powers (Muhammad is not the father of any of your men. The making of the last Prophet, ch. 8, à paraître) ».

9. AMARI Michele, «Bibliographie primitive du Coran. Extrait tiré de son mémoire inédit sur la chronologie et l'ancienne bibliographie du Coran », éd. par Hartwig Derenbourg. Centenario della nascita di Michele Amari. Scritti di filologia e storia araba; - di geografia, storia, diritto della Sicilia medievale; - studi bizantini e giudaici relativi all'Italia meridionale nel medio evo; - documenti sulle relazioni fra gli stati italiani ed il Levante, Palermo, Stabilimento Tipografico Virzì, 1910, 2 vol., p. $1-22$.

10. Le sujet du prix est mentionné dans les "actes académiques du $1^{\mathrm{er}}$ janvier 1857 au 31 décembre 1860. $§ 3$. Prix décernés et prix proposés par l'académie. Sujets et jugements des concours depuis l'année 1857 jusqu'à l'année 1860", Mémoires de l'Institut Impérial de France, Académie des Inscriptions et belles-lettres, vol. 23, 1868, p. 127-128. 
11. DÉROCHE François - NOJA NOSEDA Sergio, Le manuscrit arabe 328 (a) de la Bibliothèque nationale de France, Lesa - Paris, Fondazione Ferni Noja Noseda - Bibliothèque nationale de France, 1998, vol. 1, XCII - 238 p. (Sources de la transmission manuscrite du texte coranique, I, Les manuscrits de style hị̆ḡā ) .

12. DUTTON Yasin, “An Early Mușhaf According to the Reading of Ibn 'Āmir”, Journal of Qur'anic Studies, 2001, vol. 3, no. 1, p. 71-89.

13. Au sujet des sept feuillets du troisième cahier, les points de départ et les conclusions de l'auteur et de D. S. Powers sont divergents. Selon F.D., « Le cahier III de la $1^{\text {ère }}$ séquence (f. P 15 à 21) qui ne compte que sept feuillets ne constitue pas une véritable exception : le texte y est en effet continu du f. 15a au f.21b et se poursuit sans lacune depuis la fin du cahier II jusqu'au début du cahier IV; le talon visible dans la seconde moitié du cahier, entre les f. P 19 et P 20, ne correspond donc à aucune perte de texte » (p.24). Bien au contraire la théorie de D. S. Powers se fonde sur l'hypothèse que le cahier irrégulier de sept feuillets a été remanié pour modifier le texte coranique du verset 176 de la sūrat al-nis $\bar{a}$, en s'écartant de la prudente conclusion soulignée dans la note de bas de page de F.D «Il ne nous est pas possible de décider si le f. P 17 a toujours été dépareillé ou non » (p. 24, note 4).

14. D. S. Powers a ignoré cette présentation par double page, c'est-à-dire le verso d'un feuillet et le recto du feuillet suivant, et la continuité de la copie du texte, en basant partiellement sa théorie sur une irrégularité de la double page, conçue dans son ouvrage comme le recto et le verso d'un feuillet. Précisément il a reconstruit le verso d'un hypothétique folio 20 qui serait constitué de 23 lignes, alors que le recto du feuillet suivant (21) est composé de 25 lignes (voir Powers, Muhammad is not the father, p. 190).

15. Voir la question d'une « identité visuelle » : Alain GEORGE, The rise of Islamic calligraphy, London, Saqi, 2010, p.52.

16. BERGSTRÄSSER Gotthelf - PRETZL Otto, Die Geschichte des Korantexts, Leipzig, Dieterich'sche Verlagsbuchhandlung, 1938, XII-351 p. mit 8 Tafeln (Geschichte des Qorâns, vol. 3).

17. BlachÈRe Régis, Introduction au Coran, Paris, Maisonneuve \& Larose, 1959, XXXIII-310, p. 196.

\section{AUTEURS}

\section{ALBA FEDELI}

Università degli Studi di Milano 